\title{
Psicoterapias humanístico-existenciales: fundamentos filosóficos y metodológicos
}

\section{Humanistic-existential psychotherapies: philosophical and methodological foundations}

RESUMEN: Se realiza un análisis de la fundamentación filosófica, teórica y metodológica de las psicoterapias que conforman la "tercerafuerza" de la psicología, desde su surgimiento hasta la concepción actual, desde el punto de vista existencial, de la ansiedad.

PALABRAS CLAVE: Existencialismo, humanismo, fenomenología, ansiedad.
ABSTRACT: Philosophical, theoretical and methodological foundations of the "third force" of Psychology are analyzed, from their origins to current theories of anxiety, viewed from an existential point of view.

KEYWORDS: Existentialism, humanism, phenomenology, anxiety.

"Lo importante no es lo que han hecho conmigo, sino lo que yo hago con lo que han hecho conmigo"

SARTRE.

\section{Introducción.}

Durante los años veinte y treinta, las escuelas del estructuralismo, el funcionalismo, el conductismo, la psicología de la Gestalt y el psicoanálisis coexistieron. Sin embargo, a mediados del siglo XX, el estructuralismo había desaparecido como escuela, y el funcionalismo y la psicología de la Gestalt habían perdido su distintivo como escuelas siendo absorbidas por otros enfoques. En los años 50 y principios de los 60 , solo el conductismo y el psicoanálisis permanecieron como escuelas de pensamiento influyentes e intactas (1).

A lo largo de la historia se ha podido constatar cómo la escuela de pensamiento, filosofía o psicología predominante parece estar determinada en gran medida por el Zeitgeist o "espíritu de la época". El surgimiento de las teorías psicológicas y psicoterapias englobadas dentro de lo que se ha dado en llamar tercera fuerza de la psicología se produce en una época, los años 60 del siglo pasado, turbulenta y convulsa en la que nuevos valores comienzan a surgir. En Estados Unidos se produce un movimiento antibelicista y continuas protestas contra la guerra de Vietnam, la figura de Martin Luther King encabeza el movimiento a favor de los derechos civiles, el movimiento hippie se rebela contra los valores tradicionales de los progenitores y de la nación. En Europa tiene lugar el 
mayo del 68 y la Primavera de Praga, etc.

En una sociedad cambiante como la descrita, muchos consideraron que el conocimiento de las personas proporcionado por el psicoanálisis y el conductismo era incompleto y distorsionado concluyendo que lo que se necesitaba era una nueva visión de la psicología que no destacara ni la importancia de la mente ni la del cuerpo, sino la importancia del espíritu humano.

Los modelos agrupados bajo el epígrafe "humanístico-existencial" nacen, como se ha dicho, como alternativa al psicoanálisis y al conductismo y por ello se denomina a este movimiento tercera fuerza de la psicología. Consideran que estas otras dos fuerzas descuidan en gran medida los atributos humanos y señalan que se carece de la información que pudiera ayudar a las personas sanas a estar más sanas, es decir, a alcanzar su mayor potencial. Los psicólogos pertenecientes a este movimiento se muestran críticos contra el psicoanálisis pues lo consideran mecanicista, impersonal, formalista, jerárquico y elitista, y contra el conductismo que definen como excesivamente cientifista, frío y distante (2). Este movimiento es en gran medida una reacción contra la adopción en la psicología clínica del método científico experimental, visto como deshumanizante y despersonalizador ofreciendo como alternativa una nueva visión del hombre aspirante a la autorrealización plena (3). Este movimiento contrasta con la mayoría de otros tipos de psicología porque al contrario que éstos no asume el determinismo a la hora de explicar la conducta humana. Más bien, como veremos con detenimiento más adelante, supone que las personas son libres para elegir su propia clase de existencia. En lugar de atribuir las causas de la conducta a los estímulos, a la genética o a las experiencias tempranas, los psicólogos que adoptan este marco consideran que la causa más importante de la conducta es la realidad subjetiva.

Algunos autores presentan los modelos humanísticos junto con los existenciales $(4,5)$, otros (6) consideran que son modelos que debieran ir separados ya que su fundamentación es diferente, distinguiendo entre fenomenológicos y existenciales (Gestalt, análisis transaccional, terapia existencial, logoterapia) y humanistas (psicoterapia centrada en la persona y experienciales) (7). Pero debido a que el límite entre unos y otros no es claro y a que comparten una serie de pricipios básicos con relación a su concepción del hombre y de cara a la intervención terapéutica, en la mayoría de los textos se agrupan ambas orientaciones sin hacer distinciones.

En 1961, pricipalmente gracias a los esfuerzos de Abraham Maslow, dos hechos marcan el nacimiento de la psicología humanista: la constitución de la American Association of Humanistic Psychology (AAHP) y la publicación del Journal of Humanistic Psychology. Un año después, J. Bugental presenta el primer manifiesto humanista en la coferencia de la APA. En los años sesenta el movimiento de la psicología humanista creció de forma notable y fue aceptada como división 32 dentro de la APA. 
ORIGINALES Y REVISIONES

Antecedentes de la tercera-fuerza de la psicología.

Con los términos "tercera-fuerza", se hace referencia más que a una escuela a un movimiento que engloba una serie de enfoques surgidos de la tradición fenomenológica-existencial europea y del movimiento norteamericano de la psicología humanista.

Los antecedentes de estos modelos se remontan a las filosofías del romanticismo y el existencialismo y al método fenomenológico. El único modo de conocer la esencia y la naturaleza, los fundamentos y los pilares básicos, tanto teóricos como técnicos, de cualquiera de los enfoques psicoterapéuticos adscritos a este movimiento es profundizando en estos antecedentes filosóficos pues ambos comparten una visión del ser humano y de la forma de adquirir el conocimiento completamente diferente a la que racionalistas, empiristas y sensacionalistas adoptaron.

En esta línea, no todos los filósofos pensaban que se podía alcanzar la verdad mediante la razón o el ejercicio del intelecto, como mantenían los racionalistas, o mediante el análisis de las ideas derivadas de la experiencia, tal y como afirmaban los empiristas y sensacionalistas ingleses y franceses. Algunos pensadores sostenían que tanto unos como otros obviaban la verdadera fuente de información válida: la propia naturaleza humana. Declaraban que los humanos no solo poseen intelecto y unas ideas derivadas de la experiencia sino que disponen también de una amplia variedad de instintos y sentimientos irracionales. Se denominó románticos a aquellos filósofos que destacaban la importancia de estos componentes irracionales de la naturaleza humana. Según ellos, la forma más adecuada de encontrar la realidad humana es mediante el estudio completo de la persona, sin limitarse a sus facultades racionales o a ideas empíricamente determinadas. Durante el período del movimiento romántico, entre finales del siglo XVIII y mediados del siglo XIX, el principio del bienestar se definía como la capacidad de vivir honestamente según la propia naturaleza de cada uno. Desconfiaban de la razón, del dogma religioso, de la ciencia y de las normas sociales como guías para la conducta humana. Para ellos, la única guía válida para la conducta de una persona eran sus sentimientos honestos. Rousseau, padre del movimiento romántico, afirmaba que dando libertad las personas harían lo mejor para ellas mismas y para los demás pues el ser humano es bueno y gregario por naturaleza. Si finalmente la gente se comportaba de forma autodestructiva o antisocial era debido a que sus impulsos naturales habían sido interferidos por las fuerzas sociales. Otros pensadores que podemos considerar románticos son Goethe y Schopenhauer

Pero los románticos no eran los únicos que se rebelaron contra el racionalismo, el empirismo y el sensacionalismo. Existía otra corriente de pensamiento que también enfatizaba la importancia del significado de la vida y la capacidad de 
cada uno para elegir libremente: el existencialismo. Para los existencialistas, los aspectos humanos de mayor consideración eran sus interpretaciones personales y subjetivas sobre la vida y las tendencias establecidas a partir de esas interpretaciones. Como los románticos, consideraban a las experiencias interiores y a los sentimientos como el camino más válido para entender el comportamiento humano. Los primeros filósofos considerados existencialistas son Kierkegaard, Nietzsche y Dostoievsky. Todos estos pensadores investigaron el significado de la existencia humana y trataron de recuperar la importancia de los sentimientos humanos, el libre albedrío y la capacidad de elección así como la individualidad y la responsabilidad personal.

\section{Fenomenología.}

En su forma más general, la fenomenología se refiere a cualquier metodología que se centre en la experiencia cognitiva mientras ocurre, sin intentar reducir esta experiencia a las partes que la componen. El objetivo general es "capturar" las "experiencias esenciales" o formas elevadas de conocimiento. La palabra fenomenología nace de dos términos griegos: Pahinomenon (aparecer, mostrarse), y logia (discurso, ciencia). Desde finales del siglo XIX la fenomenología ha intentado resolver las siguientes cuestiones (8):

- Cómo se relacionan la conciencia y sus contenidos con el mundo externo.

- Cómo se pueden distinguir los fenómenos mentales de los físicos.

- Cómo se pueden distinguir los fenómenos mentales entre sí.

El origen del método fenomenológico es en gran medida una consecuencia del gran debate sobre el método que tuvo lugar durante la segunda mitad del siglo XIX, sobre todo en Alemania. Debate que giraba en torno a las similitudes y diferencias entre las Ciencias del Espíritu y las de la Naturaleza. El debate, en el que participaron pensadores de la talla de Brentano, Dilthey, Droysen, Eucken, Ebbinghaus, Freud, Husserl, Stuart Mill, Windelband y Wundt, se polarizó en torno a la distinción epistemológica establecida por Droysen y elaborada luego por Dilthey, esto es, entre las operaciones de explicar y comprender (9). Es fácil deducir la importancia de esta diferenciación pues plantea abiertamente la posición epistemológica de la psicología o, dicho de otro modo, de cómo obtenemos el conocimiento. Las ciencias del espíritu aprehenderían los hechos espirituales (humanos) de un modo real, inmediato y completo. Este método de aprehender el objeto es un autoconocimiento (10).

Retomando la distinción fundamental, es decir, que la naturaleza habría que 
ORIGINALES Y REVISIONES

explicarla pero la vida humana comprenderla, la pregunta es si los rasgos distintivos del comportamiento humano son asumibles por una epistemología inspirada en las ciencias de la naturaleza o si, por el contrario, exigen una metodología distinta, más adecuada al objeto de estudio, es decir, a la condición humana. Décadas más tarde. Camus (11) afirmaría que "la fenomenología se niega a explicar el mundo, quiere solamente ser una descripción de lo vivido".

El antecedente más inmediato de Husserl, matemático en el que se sitúa el origen de la fenomenología cuando en 1900 publica Investigaciones lógicas, es Brentano. El concepto más influyente introducido por Brentano es el de intencionalidad con el que hacía referencia a que cada acto mental se refiere a algo exterior a sí mismo. Por ejemplo, se juzga, se recuerda o se ama algo. Para estudiar los actos mentales y la intencionalidad empleó una forma de introspección a la que denominó fenomenológica y que es diferente de la introspección empleada por Wundt y Titchener, fundadores del voluntarismo y el estructuralismo respectivamente, pues el objetivo de Brentano era aprehender una experiencia psicológica íntegra y con significado en lugar de los elementos del pensamiento tomados aisladamente.

En Investigaciones lógicas, Husserl realiza una crítica y refutación radical al psicologismo, que al principio había defendido. Por influencia de Frege, Husserl llegó a convencerse de que el psicologismo o reducción de la verdad a los actos psicológicos que la descubren, convertía a la ciencia en imposible pues si la verdad se reduce a los actos psicológicos que la descubren, cabe efectivamente la posibilidad de que con otros actos psicológicos la verdad sea otra y entonces la ciencia no tendría criterios de verdad y se reduciría a convenciones. Este es el punto de partida de la fenomenología, la crítica al psicologismo y la afirmación de que las verdades de la matemática y de la lógica son distintas a las verdades de la psicología, pues las primeras descubren verdades de derecho y la segunda verdades de hecho, las verdades de derecho no pueden variar mientras que las de hecho, las de la psicología, sí (12).

Husserl escribe en 1913 Ideas para una fenomenología pura y una filosofía fenomenológica, obra fundamental que marcará un antes y un después en el empleo de este método, el cual persigue una doble finalidad: por un lado, mostrar el fundamento de cómo conocemos; por otro, detectar ciertas estructuras esenciales o "experiencias esenciales" en la conciencia, comunes a todos los seres humanos, mediante facultades como la intuición y la empatía. De esta manera, las descripciones en fenomenología solo pueden aplicarse a las experiencias subjetivas.

Husserl fue discípulo de Brentano y aceptó el concepto de intencionalidad propuesto por éste. Sin embargo fue más allá pues consideró que la intencionalidad producía solo un tipo de conocimiento, el que la persona obtenía cuando se dirigía a su entorno, pero obviaba el conocimiento procedente del propio interior, 
es decir, de lo que le aparece al sujeto. Ambos tipos de estudio utilizaban la introspección, el primero para examinar los actos mentales con los que abarcamos el mundo físico y el segundo para examinar toda la experiencia subjetiva tal y como se produce, sin necesidad de relacionarla con nada más. Con esta distinción Husserl diferencia entre el acto y el objeto del mismo, empleando para referirse a este último el término vivencia. Así, para Husserl, existían dos tipos de introspección: una centrada en la intencionalidad y otra centrada en cualquier proceso que experimentaba subjetivamente una persona. Por ejemplo, el primer tipo se preguntaría qué objeto externo intenta ver el acto de ver, el segundo se concentraría en una descripción de la experiencia de ver. Al segundo tipo de introspección Husserl lo denominó fenomenología pura.

Ideas para una fenomenología pura y una filosofía fenomenológica se compone de cuatro partes. Es en la segunda parte en la que Husserl expone la esencia del método fenomenológico cuando nos habla de la epojé, que significa literalmente "detenerse". Entendiendo epojé como neutralización, lo que Husserl plantea es que para hacer fenomenología y "volver a las cosas mismas", es necesario no participar, neutralizar o "poner entre paréntesis" todos los elementos de la vida humana consciente en los que de ordinario nos vemos inmersos. Solo mediante esta neutralización de la actitud natural, esto es, la neutralización de nuestro compromiso, ejecución y participación en todo lo que conforma la vida humana consciente o tesis cogitativas, utilizando la terminología husserliana, se alcanzaría la actitud fenomenológica (reflexiva) que requiere este método para poder centrarse en la vida subjetiva y reflexionar acerca de ella.

Cuando se emplea el término fenómeno, aunque Husserl prefiere el de vivencia, para describir un suceso mental, se refiere a la experiencia intacta, entera y con significado y no a los fragmentos de experiencias conscientes como las sensaciones aisladas. La conclusión que se puede extraer de la elaboración de Husserl es que los métodos de las ciencias naturales son inadecuados para el estudio de los fenómenos mentales o vivencias. No negaba que la psicología experimental fuera posible pero sí afirmaba que debía estar precedida de un análisis fenomenológico cuidadoso y riguroso. Es decir, resultaría prematuro realizar experimentos sobre la percepción o la memoria sin conocer previamente la esencia o naturaleza última de estos procesos. El objetivo último de Husserl no era otro que describir las esencias mentales por las que los hombres se experimentan a sí mismos, al mundo y a otros hombres mediante la información exacta de lo que aparecía en la conciencia de un modo neutral tras la adopción de esa actitud reflexiva mencionada más arriba.

En muchos textos se menciona que la publicación en 1913 de la Psicopatología General de Jaspers significó la introducción del método fenomenológico de Husserl en la psicopatología. Sin embargo, otros autores, como Berrios (13), sos- 
ORIGINALES Y REVISIONES

tienen que Jaspers realiza un uso idiosincrásico del término fenomenología que no está en la línea husserliana. Diversos estudios han llamado la atención sobre la escasa evidencia de que la fenomenología husserliana tuviera una influencia relevante en Jaspers señalando a su vez que son otros autores, como Kant, Dilthey y Weber, los que van a influir en el primero, sobre todo en las dicotomías forma/ contenido y explicación/comprensión (14).

Como señala Castilla del Pino (15), Jaspers, antes que del método fenomenológico, se vale de dichas dicotomías para, en el nivel de los fenómenos aislados, poder explicar lo formal y comprender el contenido. El término fenomenología en la obra de Jaspers hay que considerarlo como un estilo y método particular de recoger información y comprenderla, método que consiste en la descripción por empatía, y que tiene el objetivo de describir tan claramente como fuera posible las diversas situaciones psicológicas tal como son experimentadas por el paciente (16). En cuanto a la consideración de que una experiencia es comprensible o incomprensible desde el punto de vista de Jaspers, consideración que fundamenta la oposición entre los dos conceptos básicos de su obra: proceso y desarrollo, habría también mucho que decir al respecto pues algunos autores señalan que cuando se tacha una experiencia de incomprensible cabe preguntarse si se ha discriminado suficientemente entre la incomprensibilidad intrínseca de determinada vivencia o la incomprensibilidad para el observador, es decir, “¿es incomprensible per se o es incomprensible para mí?" (17).

Aunque resulta muy difícil establecer una diferenciación clara entre los conceptos de los que estamos hablando debido al solapamiento de significados y a que los autores los utilizan, como venimos viendo, en sentidos diferentes, se tiende a considerar que, aparte de la fenomenología descriptiva a la que se ha hecho alusión, existe otra versión de la fenomenología aplicada a la clínica: la fenomenología comprensiva. El objetivo de esta versión es el que resulta más congruente con las psicoterapias englobadas bajo el epígrafe de humanísticoexistenciales pues el objetivo de la misma es comprender el trastorno que está presentando la persona de acuerdo a sus circunstancias (contexto o marco de referencia). Aquí comprender sería un sinónimo de captar el sentido de la experiencia subjetiva y de las conductas observables en relación con las circunstancias personales concretas e inmediatas y el marco social de fondo en el que está inmerso el sujeto. Lo relevante en la labor clínica es captar el sentido (significado y función) del trastorno. La fenomenología comprensiva clásica ha descrito ciertas formas de la experiencia trastornada que permiten comprender el sentido de los síntomas. Aunque se trate de trastornos, no por ello las experiencias correspondientes dejan de tener su organización o "estructura" interna (18). 
Características comunes de los enfoques que integran el movimiento humanista o tercera fuerza.

Los principios básicos que comparten los modelos humanístico-existenciales aparecen recogidos en la declaración con que se constituyó la American Association of Humanistic Psychology en el año 1961, donde destacan los aspectos fundamentales de la concepción del ser humano:

- Autonomía personal y responsabilidad social.

- Autorrealización.

- Orientación hacia metas y búsqueda de sentido.

- Los seres humanos son únicos, tanto entre sí como respecto a otros animales por lo que lo aprendido a partir de otras especies es irrelevante para comprender a los primeros.

- Concepción global del ser humano.

Pese a la confluencia de diferentes posiciones teóricas, técnicas particulares y un número importante de autores (Maslow, Rogers, Kelly, Laing, Perls, May, Berne, Frankl, Lowen, etc), es posible extraer un determinado número de características comunes y compartidas por estos enfoques $(19,20)$ :

- La conducta humana es intencional. La búsqueda de sentido y las motivaciones axiológicas (libertad, dignidad), y no solo las materiales, son aspectos fundamentales del ser humano.

- El comportamiento de una persona está determinado principalmente por la percepción que tiene de sí misma y del mundo. Las personas crean la realidad a partir de sus percepciones idiosincrásicas. Solo se puede comprender a una persona si se es capaz de percibir las cosas como ella las percibe.

- Se concede poca importancia a la historia del cliente. Se hace hincapié en la experiencia inmediata y las emociones. El foco de la terapia rara vez de aleja de lo que está sucediendo durante la sesión. Se considera fundamental el presente que vive la persona.

- Solo se puede comprender a una persona cuando nos podemos situar en su lugar para percibir el mundo desde ella misma. De este modo, se rechaza el concepto de enfermedad mental y las etiquetas diagnósticas, asumiendo que todo comportamiento humano es normal si se mira desde el punto de vista de la persona afectada.

- Se da más importancia a la actitudes del terapeuta que a las técnicas concretas. La relación entre cliente y terapeuta se considera el medio único o fundamental a través del cual se consigue la autorrealización.

- El ser humano es superior a la suma de sus partes y debe ser concebido globalmente. 
ORIGINALES Y REVISIONES

- La conducta humana se realiza en un contexto interpersonal.

- La persona es considerada en sí misma como un sujeto independiente y pensante, plenamente responsable de sus actos y capaz de planear, elegir y tomar decisiones respecto a su conducta.

- Cada persona posee de forma innata un potencial de crecimiento o una tendencia a desarrollarse y convertirse en un individuo maduro. Este supuesto lleva a definir a la persona como un organismo básicamente bueno y orientado hacia metas positivas.

\section{Terapia existencial.}

Tanto esta terapia como las encuadradas dentro de este movimiento toman la perspectiva de la fenomenología y el existencialismo. Esta inspiración filosófica no sirve tanto para desarrollar teorías o modelos del funcionamiento psicológico como para plantear las cuestiones acerca de la naturaleza del ser humano y de las condiciones de la existencia. Naturaleza y condiciones que tienen que ver con la ansiedad, la tristeza, la soledad, la desesperación, la libertad, la alienación $y$, en definitiva, con el sentido de la vida.

El estudio de la filosofía y de la historia de las ideas es necesario para entender, en toda su magnitud, la naturaleza y pretensiones tanto de la terapia existencial como del resto de enfoques terapéuticos que comparten con la primera la concepción del ser humano (figura 1).

La terapia existencial aborda los problemas con relación a ciertas preocupaciones básicas de la vida y ofrece un procedimiento práctico al respecto. Se define este tipo de psicoterapia como un método de relación interpersonal y de análisis psicológico cuya finalidad es promover un conocimiento de sí mismo y una autonomía suficientes para asumir y desarrollar libremente la propia existencia (21). La noción central es la de proyecto existencial. El objetivo de la terapia es analizar ese proyecto y su modificación. La terapia no pretender cambiar la realidad externa, física o social, sino la percepción que tiene la persona sobre las cosas. Su objetivo fundamental es recuperar al ser humano para su autoposesión y autodeterminación (tabla 1). Desde esta perspectiva los trastornos y la psicopatología son formas inauténticas de existencia, estancamientos o vacíos existenciales, defensas o negaciones, renuncias o pérdidas de libertad (22). 
El ejemplo de la Logoterapia.

Viktor Frankl (1905-1997) fue Catedrático de Psiquiatría y Neurología en la Universidad de Viena y director de la Policlínica Neurológica Vienesa durante 25 años. Durante la II Guerra Mundial permaneció prisionero en Auschwitz, Dachau y otros campos de concentración nazis. Esta experiencia, a la que se suma el asesinato de sus padres, de su hermano y de su mujer, le llevaron a sistematizar sus reflexiones acerca del sentido de la vida y, aplicándolas a la psicoterapia, creó su propio enfoque: la logoterapia.

Etimológicamente el término elegido por Frankl para denominar a su enfoque viene de la palabra griega logos que equivale a "sentido", "significado" o "propósito". La logoterapia, considerada por muchos como la Tercera Escuela Vienesa de Psicoterapia (la primera y la segunda vendrían representadas por Freud y Adler), se centra en el sentido de la existencia humana y en la búsqueda de ese sentido por parte del hombre. "Vos buscás algo que no sabés lo que es", escribe Cortázar en Rayuela (23), esta búsqueda es la que abordan las terapias existenciales en general y la logoterapia en particular.

De acuerdo con la logoterapia, la primera fuerza motivante del hombre es la lucha por encontrarle un sentido a su propia vida, este es el motivo de que Frankl aluda constantemente en su obra a la voluntad de sentido. Cuando el hombre malogra su voluntad de sentido, es decir, cuando se produce un vacío existencial por la pérdida de sentido en la vida se puede producir un tipo de neurosis para la que Frankl acuña el término noógena. Este tipo de neurosis procede de la frustración existencial mencionada, esto es, la pérdida de sentido. La labor del terapeuta consiste por lo tanto en ayudar al sujeto a encontrar o restablecer el sentido de su vida "porque la vida es potencialmente significativa hasta el último momento, hasta el último suspiro, gracias al hecho de que se pueden extraer significados hasta del sufrimiento" (24).

Frankl plantea que el ser humano tiene la capacidad de autodistanciarse $y$ autotrascender. Estas capacidades son potenciadas en la terapia mediante dos técnicas innovadoras introducidas por Frankl y empleadas posteriormente por enfoques muy diversos (cognitivos, sistémicos), a saber: la intención paradójica y la derreflexión (25).

Concepción existencialista de la ansiedad.

La presentación ofrecida de la logoterapia es el punto de partida idóneo para retomar algunas de las aportaciones conceptuales y teóricas ofrecidas por 
ORIGINALES Y REVISIONES

el existencialismo, sobre todo en lo que a la libertad de elección y la ansiedad se refiere.

Una de las máximas de esta corriente de pensamiento es que los humanos eligen la naturaleza de su propia existencia. Es esta capacidad de elección o libre albedrío la que nos responsabiliza de nuestra propia vida y más aún, según Heidegger, fuente de culpa en el caso de no ejercitarla. En otras palabras, si no ejercitamos nuestra propia libertad experimentaremos culpa. Todo el mundo podría reducir la culpabilidad intentando llevar una vida auténtica, es decir, reconocer y vivir siendo fieles a nuestra habilidad para elegir nuestra propia existencia, esta fidelidad y habilidad nos permitirá vivir de un modo auténtico.

La ansiedad, desde el punto de vista de Heidegger, es una parte necesaria de la vida auténtica. La angustia se concibe ontológico-existencialmente como el fenómeno originario (26). Una de las razones de esta ansiedad es que las personas auténticas, es decir, aquellos que no viven conforme dicta la sociedad y no dejan su libertad en manos de los otros, están siempre experimentando con la vida, compitiendo y logrando objetivos. Entrar en lo desconocido causa parte de la ansiedad asociada a la vida auténtica. Otra de las razones que esgrime Heidegger para argumentar que ejercitar la libertad causa ansiedad es que le hace a uno responsable de las consecuencias de esta elección. El individuo libre no puede culpar ni a Dios, ni a los padres, ni a los genes, ni a la sociedad ni a ninguna otra cosa por lo que ha llegado a ser.

En el análisis de la angustia se han de considerar tres aspectos: el ante qué, el qué y el porqué se tiene angustia. El ante qué es el mundo en su falta de significado, manisfestándose así inseguro e inhóspito. El mundo ya no puede ofrecer nada ni tampoco la coexistencia con los otros. Recordemos que el término adoptado por Heidegger Dasein quiere decir, literalmente, "ser" (sein) "allî" $(D a), \mathrm{y}$ Heidegger describe la relación entre una persona y el mundo como "ser-en-el. mundo" e indica que una persona y el mundo son inseparables. Por otro lado, este mundo está también poblado de otros hombres siendo la existencia de una persona también coexistencia con esos otros. Entonces, anté qué se tiene angustia, ante lo desconocido y la incertidumbre o, como dice Sartre irónicamente: “QQué puede temerse de un mundo tan regular? Creo que estoy curado" (27).

El qué de tener angustia es el desasosiego y la desazón de quedar devuelto a sí mismo y aislado. El porqué de la angustia es la indeterminación en la que queda el Dasein como ser posible que tiene ahora que determinarse a sí mismo. Esta angustia, como decía más arriba, revela la libertad del Dasein. Yendo un poco más allá, lo único seguro en medio de la incertidumbre es la muerte, por lo tanto la angustia acaba por evidenciar la verdadera condición del Dasein de serpara-la-muerte. Así, otro de los requisitos previos propuestos por Heidegger para vivir una vida auténtica es la necesariedad de afrontar que algún día moriremos 
pues es la muerte la que da sentido a la vida.

La perspectiva de la angustia de Heidegger deriva de la planteada por Kierkegaard solo que la primera es una versión secular de la segunda. A pesar de esta diferencia, ambas vienen a decir, como lo harán las posteriores concepciones de Binswanger, Sartre, Frankl, May, Fromm y la mayoría de los existencialistas, que podemos ejercitar nuestra libertad y experimentar ansiedad o no ejercirtarla y sentirnos culpables.

Como propone May, la ansiedad neurótica sería aquella que no conduce al desarrollo personal porque es el resultado del miedo a la libertad pues la persona vive su vida de tal modo que reduce o elimina su capacidad de elección y libertad personal. Kierkegaard llamó a esta situación neurótica encerramiento. La autoalienación ocurrirá cuando la gente acepta, como algo propio, los valores dictados por la sociedad en detrimento de los suyos propios. Este estado de autoalienación no termina con el sentimiento de culpa sino que a éste le acompañan la desesperación y la apatía.

Por otro lado y como es fácil de vislumbrar, a esta ansiedad neurótica le acompaña la ansiedad normal que aparece inevitablemente, y como se viene señalando, cuando se decide tener una vida auténtica.

Binswanger mediante el análisis existencial y Frankl con la logoterapia pretenden, de un modo genérico, ayudar a la persona a encontrar el sentido de su propia existencia. Binswanger habla de tres modos diferentes de existencia, a saber: el Umwelt o mundo físico, el Mitwelt o mundo de las relaciones con los otros y el Eigenwelt o mundo interno. Para entender a la persona y así poder brindarle ayuda, sería necesario entender los tres modos de existencia. Uno de los conceptos más importantes introducidos por Binswanger es el de Weltanschauung o diseño del mundo. Si el diseño del mundo que hace una persona es inefectivo pues le produce demasiado miedo, ansiedad o culpa, será tarea del terapeuta hacerle ver que hay otros modos de existir con respecto al mundo, a los otros y a uno mismo. Del mismo modo, la logoterapia pretende ayudar al sujeto a encontrar el sentido de su vida, a hallar el significado perdido. La mayoría de los existencialistas subrayan la frase de Nietzsche "lo que no me mata, me hace más fuerte” pues esta fuerza proviene de encontrar el significado incluso a una experiencia negativa.

Heidegger, y posteriormente Binswanger aceptando la visión del primero, puso límites a la libertad personal. Argumentaba que habíamos sido lanzados a nuestro $D a$ o allí por circunstancias que están fuera de nuestro control. Este lanzamiento determina las condiciones bajo las cuales ejercitamos nuestra libertad. Según Heidegger, todas las personas son libres, pero las circunstancias bajo las cuales esta libertad se ejercita varían. El lanzamiento aporta el contexto para la propia existencia. No se trata pues de negar la influencia de la herencia, de las 
ORIGINALES Y REVISIONES

experiencias de las primeras etapas o del medio social que nos rodea sino de intentar transformar las circunstancias al ejercitar el libre albedrío.

Frankl considera, al igual que el resto de existencialistas, que "la frustración existencial no es en sí misma ni patológica ni patogénica. La preocupación, o la desesperación, por encontrarle a la vida un sentido valioso es una angustia espiritual, pero en modo alguno representa una enfermedad. Bien pudiera suceder que si se interpreta la angustia en términos de enfermedad, el psiquiatra se sienta inclinado a enterrar la frustración existencial de su paciente bajo un tratamiento de drogas tranquilizantes”.

\section{Conclusiones.}

Las escuelas contra las que los enfoques adscritos a esta tercera fuerza de la psicología, adoptando los postulados filosóficos románticos y existenciales así como el método fenomenológico, se rebelaron, esto es, el psicoanálisis y el conductismo, hoy en día no son concebidas como entonces. En el caso del psicoanálisis es de sobra conocida la diáspora de escuelas y tendencias surgidas a partir del psicoanálisis ortodoxo freudiano. Escuelas unas y tendencias otras que, a pesar de sus diferencias, tienen en común la discrepancia con la teoría ortodoxa. Si miramos este devenir con un poco más de distancia es posible apreciar que, en su conjunto, estos cambios cuestionan por sí mismos lo que vino a cuestionar la tercera-fuerza, es decir, la concepción del ser humano. En cuanto al conductismo, más de lo mismo. Desde que Watson pronunció en 1913 su conferencia "Psicología vista por un conductista” y Skinner aplicó el análisis experimental de la conducta a problemas de relevancia clínica y social creando el análisis conductal aplicado (28), el estado de las cosas ha cambiado mucho. A lo largo de las décadas se han ido desarrollando otros enfoques que, aunque basados en la aplicación de las técnicas del aprendizaje y en la metodología experimental, han introducido otras variables relevantes. Se han ido ampliando de esta manera tanto las variantes de la modificación de conducta como las técnicas empleadas. Actualmente los enfoques coexistentes dentro de la modificación de conducta son el análisis conductual aplicado, el enfoque E-R neoconductista, el enfoque del aprendizaje social desarrollado por Bandura y el enfoque cognitivo-conductual. Si se han ido introduciendo estas variables es porque paralelamente la investigación básica ha hecho su trabajo centrándose a partir de los años 60 en esas variables cognitivas de las que hablaba. Del mismo modo existen terapias cognitivas, sin el apellido de "conductual". Alejándonos lo suficiente es fácil apreciar cómo con el conductismo ocurre lo mismo que con el psicoanálisis, es decir, cambia el modo de con- 
cebir al ser humano y por lo tanto cambia el modo de concebir sus alteraciones, sus problemas y el abordaje de los mismos.

En el momento actual esta tercera-fuerza puede que no emergiera en contraposición ni al psicoanálisis ni al conductismo sino que probablemente se rebelaría en contra del reduccionismo biologicista al que tiende nuestra disciplina si no es que está inmersa ya en el mismo. Dicha concepción de nuestro objeto de trabajo y estudio tiene como consecuencia el empleo indiscriminado de psicofármacos, que al fin y al cabo presenta la misma falta de consideración hacia la persona que en su día, y según los planteamientos que a lo largo del texto se han expuesto, presentaron conductismo y psicoanálisis. Como señala Breggin (29), y nosotros subrayamos, "la cuestión es que la psicofarmacología ha sustituido valores humanos como la responsabilidad de la propia vida por la fe en "desequilibrios bioquímicos" o alteraciones cerebrales que nada tienen que ver con lo que realmente le pasa a la gente y en supuestos expertos que nada entienden de asuntos humanos".

Figura 1

Desarrollo y líneas de influencia del existencialismo y la fenomenología
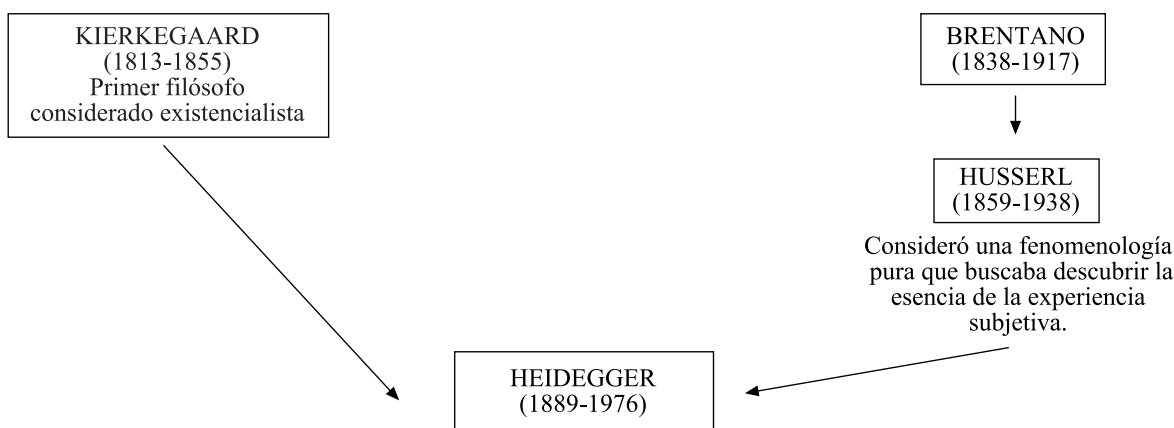

Su trabajo se considera el puente entre la filosofía existencial y la psicología existencial. Empleó el método fenomenológico para analizar la totalidad de la existencia humana.Con el término Dasein indica que el mundo y el sujeto son inseparables. Describe la relación entre el mundo y el sujeto como "ser-en-el-mundo". Dasein singnifica existir en el mundo y existir es convertirse en algo diferente constantemente, cambiar. Los hombres eligen la naturaleza de su propia existencia.

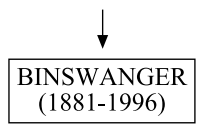

Aplicó la fenomenología a la psiquiatría. Su objetivo fue integrar los escritos de Heidegger y Husserl con la teoría psicoanalítica (estudió psiquiatría con Bleuler y psicoanálisis con Jung. Fue uno de los psicoanalistas freudianos en Suiza). Adoptando la noción de Dasein de Heidegger denominó a su enfoque sobre la psicoterapia Daseinanálisis o análisis existencial.

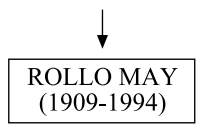

Introduce el existencialismo de Heidegger en la psicología americana. Fue el primer responsable de reincorporar la filosofía existencial europea a la psicología estadounidense. 
ORIGINALES Y REVISIONES

Tabla 1

Preocupaciones existenciales, horizonte terapéutico y técnicas utilizadas (modificado de Pérez-Álvarez, 1996)

\begin{tabular}{|l|l|l|}
\hline $\begin{array}{l}\text { Preocupaciones } \\
\text { Existenciales }\end{array}$ & $\begin{array}{l}\text { Horizonte de } \\
\text { Sentido }\end{array}$ & \multicolumn{1}{c|}{ TÉCNICAS } \\
\hline $\begin{array}{l}\text { Angustia ante la } \\
\text { muerte }\end{array}$ & $\begin{array}{l}\text { Adquisición de } \\
\text { fortaleza }\end{array}$ & $\begin{array}{l}\text { Desidentificación: confrontar la muerte cara a un cambio personal. } \\
\text { Desensibilización: familiarizarse y acostumbrarse a la muerte. } \\
\text { Reconstrucción situacional: } \text { enfatizar las condiciones situacionales en } \\
\text { lugar de las emocionales. El sujeto imagina un suceso estresante en } \\
\text { las peores condiciones y después lo imagina en circunstancias más } \\
\text { agradables, con el fin de que descubra la importancia de las } \\
\text { circunstancias, al reconstruirlas, a la hora de valorar sus acontecimientos } \\
\text { vitales, dando relevancia a la interacción que desarrolla la persona con } \\
\text { su ambiente circundante. }\end{array}$ \\
\hline Asumir la libertad & $\begin{array}{l}\text { Toma de } \\
\text { responsabilidad }\end{array}$ & $\begin{array}{l}\text { "Hacerse cargo". } \\
\text { Confrontar sustituyendo el no puedo por el no quiero. } \\
\text { Desarrollar la voluntad. }\end{array}$ \\
\hline $\begin{array}{l}\text { La incomunicación } \\
\text { vida sentido de la }\end{array}$ & $\begin{array}{l}\text { Confrontar la } \\
\text { soledad }\end{array}$ & $\begin{array}{l}\text { Autodistancia- } \\
\text { Idienticar y comprender lo que hace por los demás. } \\
\text { Aprender a estar en soledad. }\end{array}$ \\
$\begin{array}{l}\text { La intención paradójica: } \text { pretende recuperar el control exagerándolo, } \\
\text { trascendencia } \\
\text { es decir, pidoendo al cliente que intente perder aún más el control en } \\
\text { lugar de luchar contra él. } \\
\text { La derreflexión: consiste en instruir al cliene para que deje de atenderse } \\
\text { a sí mismo y se concentre en el ambiente externo como determinante } \\
\text { del desarrollo de la persona. }\end{array}$ \\
\hline
\end{tabular}


ORIGINALES Y REVISIONES

\section{BIBLIOGRAFÍA:}

(1) Hergenhanh, B.R. Introducción a la historia de la psicología. Madrid, Thomson Paraninfo, 2001 .

(2) Cushman, P. "Psychotherapy to 1992: A historically situated interpretation". En D. K. Freedheim (de). History of psychotherapy. A century of change. Washington: American Psychology Association, 1992.

(3) FeIXAs, G y Miró, M.T. Aproximaciones a la psicoterapia. Una introducción a los tratamientos psicológicos. Barcelona, Paidós, 2000.

(4) Martorell, J. Psicoterapias: escuelas y conceptos básicos. Madrid, Pirámide, 1996.

(5) Rodríguez-Naranjo, C. De los principios de la psicología a la práctica clínica. Madrid, Pirámide, 2000.

(6) Pérez-Álvarez, M. Tratamientos psicológicos. Madrid, Universitas, 1996.

(7) Rosa Alcázar A.I, Olivares Rodríguez J y Méndez Carrillo F.X. Introducción a las técnicas de intervención y tratamiento psicológico. Madrid, Pirámide, 2004.

(8) Berrios, G.E. "What is phenomenology? A review". Journal of the Royal Society of Medicine, 1989, 82, pp. 425-428.

(9) PinIlLos DíAz, J.L. "Qué es psicohistoria”. En Romero Cuadra, J.L y Álvaro Vázquez R. (ed). Psicópolis. Paradigmas actuales y alternativas en la psicología contemporánea. Barcelona, Kairós, 2005.

(10) Luque, R y Villagrán, J.M. Psicopatología descriptiva: nuevas tendencias. Madrid, Trotta, 2000.

(11) Camus, A. El mito de Sísifo. Madrid, Alianza Editorial, 2006.

(12) San Martín Sala, J. "Psicología y fenomenología". En Romero Cuadra, J.L y Álvaro Vázquez R. (ed). Psicópolis. Paradigmas actuales y alternativas en la psicología contemporánea. Barecelona, Kairós, 2005.

(13) BERRIOs, G.E. "Phenomenology, Psychopathology and Jaspers: a conceptual history". History of Psychiatry. 1992, 3, pp: 303-327.

(14) Walter C. "Karl Jaspers y Edmund Husserl, I, II and III. Philosophy, Psychiatry and Psychology" 1994-1995; 1: 117-134, 245-265; 2: 65-82.

(15) Castilla del Pino, C. "Crítica a la razón psicopatológica". En Castilla del Pino, C y Ruíz Vargas, J.M. (de). Aspectos cognitivos de la esquizofrenia. Madrid, Trotta, 1991.

(16) Fish. Psicopatología Clínica. Signos y síntomas en Psiquiatría. Madrid, Emalsa, 1986.

(17) Castilla del Pino, C. Criterios de objetivación en psicopatología (dir). Madrid, AEN, 1989.

(18) González Pardo, H y Pérez Álvarez, M. La invención de los trastornos mentales. Madrid, Alianza Editorial, 2008.

(19) Bernstein, D. A y Nietzel, M. T. Introducción a la psicología clínica. México, McGrawHill, 1982.

(20) Carrobles, J. A. "Paradigmas o modelos sobre las anormalidades psicológicas: El modelo médico o fisiológico, el modelo psicodinámico, el modelo fenomenológico o humanista y el modelo estadístico". En J. A. Carrobles (de), Análisis y modificación de la conducta II. Madrid, UNED, 1985.

(21) Villegas, M. "El análisis existencial: cuestiones de método". Revista de Psiquiatría y Psicología Humanista, 1988, 25, pp. 55-70.

(22) Villegas, M. La psicoterapia existencial. Tesis doctoral no publicada. Universidad de Barcelona, 1981. 
(23) CoRtÁzar, J. Rayuela. Madrid, Cátedra, 2007.

(24) FrankL, V. La voluntad de sentido. Conferencias escogidas sobre logoterapia. Barcelona. Herder, 1994.

(25) FrankL, V. El hombre en busca de sentido. Barcelona, Herder, 2004.

(26) HeIDEGGer, M. Ser y tiempo. Madrid, Trotta, 2003.

(27) SARtre, J.P. La naúsea. Madrid, Alianza Editorial, 1990.

(28) Olivares, J, Méndez Carrillo F.X, y Maciá, D. Tratamientos conductuales en la infancia y adolescencia: bases históricas, conceptuales y metodológicas. Situación actual y perspectivas futuras. Madrid, Pirámide, 1997.

(29) P.R. BREGGIN. "Psychopharmacology and human values". Journal of Humanistic Psychology.2003, 43, 2, pp. 34-49.

* Cristina Tobías Imbernón, PIR Hospital Universitario de Canarias (La Laguna, Santa Cruz de Tenerife); Jose García-Valdecasas Campelo, psiquiatra Hospital Universitario de Canarias (La Laguna, Santa Cruz de Tenerife).

Correspondencia: Cristina Tobías Imbernón. Hospital Universitario de Canarias. Ofra, s/n. La Cuesta, 38320 La Laguna. Santa Cruz de Tenerife; Correo electrónico: ctimbernon@ hotmail.com.

** Recibido: 15/04/2009 